\title{
Adherencia farmacológica de pacientes con diabetes mellitus en un programa de nefroprotección: una responsabilidad compartida
}

Medication adherence of patients with diabetes mellitus in a nephroprevention program: a shared responsibility

\author{
Nury Ximena Trejo-Bastidas ${ }^{1 \otimes}$, Jairo Jesús Eraso-Paredes ${ }^{2}{ }$, Heidy Johanna Contreras- \\ Martínez ${ }^{3}$ CvLAC
}

Fecha correspondencia:

Recibido: noviembre 15 de 2018. Revisado: marzo 29 de 2019. Aceptado: noviembre 6 de 2019.

Forma de citar:

Trejo-Bastidas NX, Eraso-

Paredes JJ, Contreras-Martínez

HJ. Adherencia farmacológica

de pacientes con diabetes

mellitus en un programa

de nefroprotección. Una

responsabilidad compartida. Rev

CES Med 2020; 34(1): 3-13.

Open access

(c) Derecho de autor

Licencia creative commons

Ética de publicaciones

Revisión por pares

Gestión por Open Journal System

DOl: http://dx.doi.org/10.21615/

cesmedicina.34.1.1

ISSN 0120-8705

e-ISSN 2215-9177

\section{Resumen}

Introducción: la diabetes mellitus tipo dos es una enfermedad crónica de gran impacto en salud pública; su mal control clínico está supeditado a la deficiente adherencia farmacológica. Los programas de nefroprotección intentan mejorar el control de esta enfermedad y una adecuada adherencia puede ser una estrategia. El objetivo del estudio fue determinar los factores asociados con la adherencia farmacológica de pacientes con diabetes en el contexto de un programa de nefroprotección. Materiales y métodos: estudio transversal analítico en 282 pacientes con diabetes mellitus tipo dos de un programa de nefroprotección en los municipios de Pasto y Túquerres en el año 2017, seleccionados mediante muestreo aleatorio, sistemático estratificado. La adherencia se midió con el test Morisky-Green. La información se obtuvo de bases de datos de las historias clínicas y mediante un cuestionario estructurado. Se efectuaron análisis descriptivo, bivariado y regresión logística binaria multivariada con fines explicativos. Las medidas de asociación fueron razones de prevalencia (RP). Resultados: la mediana de la edad fue de 67 años, 33 \% fueron hombres y la proporción de adherencia del $68 \%$; los factores que se asociaron independientemente fueron, ser mujer RP: 1,25 (IC $95 \%$ : 1,02-4,07), hemoglobina glicosilada entre 6 y 6,9 \% RP: 1,66 (1,37-11,80), no uso de insulina RP: 1,36 (1,03-8,52), empleo de solo metformina RP: $1,76(1,76-10,15)$ y consulta por medicina interna RP: 1,19 (1,19-4,78). Conclusión: la adherencia farmacológica está influenciada por múltiples factores que no sólo dependen del paciente, sino que involucran también a los profesionales de salud, el tipo de medicación y su disponibilidad, además de asociarse con el control glucémico.

Palabras clave: Diabetes mellitus tipo 2; Adherencia al tratamiento; Nefroprotección; Insuficiencia renal crónica.

Comparte

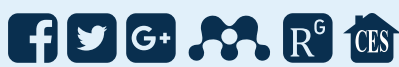

\section{Abstract.}

Introduction: The type two diabetes is a chronic disease of great impact on public health. Its deficient clinical control is subject to poor medication adherence. Nephroprevention programs seek to improve the control 


\section{Sobre los autores:}

1. Médica general asistencial en COOEMSSANAR IPS,

Pasto, Colombia. Magíster en Epidemiología. Universidad CES, Medellín, Colombia. Grupo de investigación: Epidemiología y Bioestadística, Universidad CES.

\section{Médico auditor en EMSSANAR SAS, Pasto, Colombia. Magíster en Epidemiología. Universidad CES, Medellín, Colombia. Grupo de investigación: Epidemiología y Bioestadística, Universidad CES.}

3. Química farmacéutica, Magister en Epidemiología. Docente en Universidad CES, Medellín, Colombia. Grupo de Investigación en Ciencias Farmacéuticas -ICIF- de Universidad CES.

Solo cerca del $50 \%$ de los pacientes con diabetes logran las metas de control, lo cual parece explicarse en gran parte, por la pobre adherencia farmacológica. of this disease and an adequate adherence may be a strategy. The aim of this study was to determine the factors associated with medication adherence in patients with diabetes in the context of a nephroprevention program. Materials and methods: A cross-sectional study was conducted among 282 patients with type two diabetes mellitus from a nephroprevention program in the municipalities of Pasto and Túquerres in 2017; they were selected by a systematic stratified random sampling. The Morisky-Green test assessed adherence. Information was obtained from databases, medical records and through a structured questionnaire. A descriptive, bivariate analysis and a multivariate binary logistic regression for explanatory purposes were performed. The association measures were prevalence ratios (PR). Results: The median age of the participants was 67 years, $33 \%$ were men and the proportion of adherence was $68 \%$. Factors that were independently associated with adherence were being a woman, PR: 1,25 (95\% Cl: 1,02-4,07), glycosylated hemoglobin between $6 \%-6,9 \%$, PR: 1,66 (1,37-11,80), not using insulin PR: 1,36 (1,03-8,52), use of only metformin PR: 1,76 $(1,76-10,15)$ and consultation by internal medicine, PR: 1,19 (1,19-4,78). Conclusion: The adherence to medication is influenced by multiple factors which not only depend on the patient, but also involve health professionals, the type of medication and its availability, as well as being associated with glycemic control..

Keywords: Type 2 diabetes mellitus; Medication adherence; Nephroprevention; Renal Insufficiency, Chronic.

\section{Introducción}

La diabetes mellitus tipo dos, en adelante mencionada como diabetes, es catalogada una de las enfermedades crónicas de mayor relevancia a nivel mundial por sus implicaciones en la salud pública. Para 2014 la Organización Mundial de la Salud (OMS) reportaba una prevalencia global de $8,5 \%$ (1). El control de la enfermedad se ha complejizado, pues a pesar de los múltiples medicamentos disponibles, solo cerca del $50 \%$ de los pacientes logran las metas de control, lo cual parece explicarse en gran parte, por la pobre adherencia farmacológica $(2,3)$.

La adherencia terapéutica es definida por la OMS como "el grado en que el comportamiento de una persona -tomar el medicamento, seguir un régimen alimentario, y ejecutar cambios del modo de vida- se corresponde con las recomendaciones acordadas con un prestador de asistencia sanitaria" (2). En diabetes, una pobre adherencia contribuye a la aparición de complicaciones agudas y crónicas que conllevan a un mayor número de hospitalizaciones y consultas $(4,5)$. La proporción de adherencia en pacientes con diabetes, a nivel mundial varía entre 9 y $93 \%(5,6)$. En Colombia, algunos estudios reportan una proporción de adherencia que oscila entre el 68 y 79 \% (7-9).

Algunas investigaciones muestran que ciertos factores socioeconómicos, clínicos y psicológicos se asocian con la adherencia; sin embargo, no se han estudiado en el contexto de un programa de nefroprotección $(6,8,10)$. Estos se crearon para brindar servicios de salud que, de forma sistemática, realicen acciones para detectar y tratar enfermedades precursoras (hipertensión arterial, diabetes mellitus) de la enfermedad renal o si ya está presente intervenir oportunamente $(11,12)$.

El objetivo del estudio fue determinar los factores sociales, demográficos, clínicos y de los servicios de salud, asociados con la adherencia farmacológica de pacientes con diabetes mellitus tipo dos, en el contexto de un programa de nefroprotección. 
La adherencia farmacológica se trató como variable dicotómica (sí/no), medida con la prueba Morisky-Green que ha sido validado para el idioma español y en diabetes.

\section{Metodología}

Estudio de corte trasversal con pacientes de un programa de nefroprotección en los municipios de Pasto y Túquerres (Nariño, Colombia) de una entidad promotora de salud, mayoritariamente del régimen subsidiado. Los pacientes incluidos en el estudio fueron mayores de edad, con diagnóstico mayor de seis meses de diabetes mellitus tipo dos, en tratamiento farmacológico, con una antigüedad en el programa mayor a un semestre. Se excluyeron aquellas personas con discapacidad cognitiva o auditiva, quienes tuvieran enfermedad renal crónica en estadio cinco, pacientes cuyo último control médico fuera de más de seis meses y mujeres gestantes.

Para el cálculo de la muestra se tuvo en cuenta una proporción del 56 \% (13); el marco muestral era de 983 pacientes en la base de datos del programa de nefroprotección, una precisión del 5 \% y un nivel de confianza del $95 \%$; para obtener una muestra de 274 participantes. El muestreo fue probabilístico, aleatorio sistemático, estratificado, por afijación proporcional según el municipio de pertenencia. Los pacientes seleccionados de la base de datos fueron contactados telefónicamente para obtener el consentimiento informado verbal y aplicarles un cuestionario estructurado, posteriormente se recolectaron los datos pertinentes de la historia clínica.

La adherencia farmacológica se trató como variable dicotómica (sí/no), medida con la prueba Morisky-Green que ha sido validado para el idioma español y en diabetes. Consta de cuatro preguntas que, adaptadas para Colombia se enuncian así: 1) ¿Se olvida tomar alguna vez los medicamentos para tratar su enfermedad? 2) ¿Es descuidado con la hora en que debe tomar la medicación? 3) Cuando se encuentra bien, ¿deja de tomar la medicación? 4) Si alguna vez le cae mal el medicamento, ¿deja de tomarlo? Si el paciente contesta "Sí" en al menos una de ellas se considera no adherente (14-16).

Las variables analizadas fueron de tipo sociodemográfico, de asistencia personal, características clínicas y de los servicios de salud, las cuales se recolectaron de fuente mixta: mediante llamada telefónica y de las bases de datos. De las historias clínicas se obtuvo información de la adherencia farmacológica, aclarando que la prueba de Morisky-Green es de obligatorio diligenciamiento por parte de los médicos en la historia clínica electrónica del programa de nefroprotección. La recolección se hizo entre noviembre de 2017 a febrero 2018, por personal previamente capacitado.

Las variables cualitativas se analizaron por medio de medidas de frecuencias absolutas y relativas; a las cuantitativas se les aplicó pruebas de normalidad, medidas de tendencia central y dispersión. En el análisis bivariado se utilizó chi cuadrado o test de Fisher en las variables cualitativas, y la prueba $U$ de Mann Whitney para las cuantitativas. Para estimar la fuerza de asociación se usaron razones de prevalencia (RP) con su respectivo intervalo de confianza de $95 \%$ (IC $95 \%$ ) y se consideró significativo un valor de $\mathrm{p}$ menor de 0,05.

Los factores independientes fueron determinados mediante regresión logística binaria con fines explicativos y para controlar posibles confusores, como la polifarmacia y el uso de insulina. Las razones de prevalencia ajustadas se calcularon a partir de los Odds Ratio de la regresión con la fórmula propuesta por Zocchetti y los intervalos de confianza con la de Miettinen (17). Los paquetes estadísticos utilizados fueron SPSS $22.0{ }^{\circledR}$ (licencia Universidad CES) y Epidat 3.1. 
La mediana de la duración de la enfermedad fue de ocho años y de la HbA1c de 6,5 La proporción de adherencia encontrada fue del $68 \%$.
Para controlar sesgos se realizó el muestreo probabilístico, se verificaron los criterios de inclusión y exclusión, los entrevistadores fueron capacitados previamente, se utilizó un cuestionario estructurado y se garantizó a los participantes la confidencialidad de la información suministrada por ellos.

El proyecto fue avalado por el comité de ética de investigaciones de la Universidad CES con el código Ae-98. La investigación se ajusta a las normas de la resolución 8430 de 1993 del Ministerio de Salud de Colombia, como una investigación sin riesgo (18).

\section{Resultados}

Se analizaron 282 pacientes. La mediana de la edad fue de 67 años (rango intercuartil -RIQ- 16), el 66,7 \% fueron mujeres, 80,9 \% residía en el municipio de Pasto, 82,3 \% provenía de zona urbana, en su mayoría vivían en estrato medio (75,9\%) o bajo (24,1\%). Con respecto a la etnia, el 99,3\% fueron mestizos; el $96 \%$ refirió vivir con familiares y el 3 \% vivían solos. El 53,5 \% estaban casados o en unión libre, el $31,6 \%$ separados o viudos y el $14,9 \%$ solteros. El $97,5 \%$ de los pacientes pertenecía al régimen subsidiado en salud. Ninguna de estas características se asoció con la adherencia (cuadro 1).

Cuadro1. Características sociodemográficas de los pacientes del estudio y su relación con la adherencia farmacológica

\begin{tabular}{|c|c|c|c|c|}
\hline \multirow{3}{*}{ Factores } & \multirow{3}{*}{$\begin{array}{c}\text { Total } \\
\text { n=282 } \\
\%\end{array}$} & \multicolumn{2}{|c|}{ Adherencia } & \multirow{3}{*}{$R P^{a}(I C 95 \%)$} \\
\hline & & Si, $n=192$ & No, $n=90$ & \\
\hline & & $\%$ & $\%$ & \\
\hline \multicolumn{5}{|c|}{ Sexo } \\
\hline Hombre & 33,3 & 29,2 & 42,2 & 1 \\
\hline Mujer & 66,7 & 70,8 & 57,8 & $1,21(1,01-1,47)$ \\
\hline \multicolumn{5}{|c|}{ Edad } \\
\hline$<60$ años & 22,7 & 21,9 & 24,4 & $0,95(0,78-1,16)$ \\
\hline 60 y más & 77,3 & 78,1 & 75,6 & 1 \\
\hline \multicolumn{5}{|c|}{ Escolaridad } \\
\hline Ninguna & 13,8 & 14,6 & 12,2 & 1 \\
\hline Primaria incompleta & 8,2 & 5,7 & 13,3 & $0,67(0,42-1,07)$ \\
\hline Primaria completa & 65,6 & 66,1 & 54,4 & $0,96(0,77-1,19)$ \\
\hline Bachiller completo & 11,0 & 11,5 & 10,0 & $0,99(0,73-1,33)$ \\
\hline Educación superior &, 4 & 2,1 & 0,0 & $1,39(1,14-1,70)$ \\
\hline \multicolumn{5}{|c|}{ Compañía a consulta } \\
\hline Nunca & 9,3 & 8,9 & 10,0 & 1 \\
\hline A veces & 56,6 & 56,5 & 56,7 & $1,04(0,77-1,40)$ \\
\hline Siempre & 34,2 & 34,3 & 33,3 & $1,05(0,77-1,43)$ \\
\hline
\end{tabular}

RP: razón de prevalencias

La mediana de la duración de la enfermedad en los pacientes fue de ocho años (RIQ: 8), y de la HbA1c de 6,5 (RIQ: 1,4). La proporción de adherencia encontrada fue del $68 \%$ (cuadro 2). 
Cuadro 2. Características clínicas de los pacientes del estudio y su relación con la adherencia farmacológica

\begin{tabular}{|c|c|c|c|c|}
\hline \multirow{3}{*}{ Factores } & \multirow{3}{*}{$\begin{array}{c}\text { Total } \\
n=282 \\
\%\end{array}$} & \multicolumn{2}{|c|}{ Adherencia } & \multirow{3}{*}{$R P^{a}$ (IC $\left.95 \%\right)$} \\
\hline & & Si, $n=192$ & No, $n=90$ & \\
\hline & & $\%$ & $\%$ & \\
\hline \multicolumn{5}{|c|}{ Hipertensión arterial } \\
\hline No & 21,3 & 20,8 & 22,2 & 1 \\
\hline Sí & 78,7 & 79,2 & 77,8 & $1,03(0,84-1,25)$ \\
\hline \multicolumn{5}{|c|}{ Dislipidemia } \\
\hline No & 26,6 & 25,0 & 30,0 & 1 \\
\hline Sí & 73,4 & 75,0 & 70,0 & $1,07(0,91-1,25)$ \\
\hline \multicolumn{5}{|c|}{$E R C^{b}$} \\
\hline Con ERC & 54,3 & 53,6 & 55,6 & 1 \\
\hline $\operatorname{Sin} E R C$ & 45,7 & 46,4 & 44,4 & $1,02(0,87-1,20)$ \\
\hline \multicolumn{5}{|c|}{$H b A 1 c^{c}$} \\
\hline$<6$ & 27,8 & 26,9 & 29,7 & $1,42(0,95-2,13)$ \\
\hline 6 a 6,9 & 39,6 & 45,7 & 26,2 & $1,69(1,16-2,48)$ \\
\hline 7 a 8,9 & 20,7 & 19,4 & 23,8 & $1,37(0,90-2,08)$ \\
\hline$\geq 9$ & 11,9 & 8,1 & 20,2 & 1 \\
\hline \multicolumn{5}{|c|}{ Uso de insulina } \\
\hline Sí & 37,2 & 32,8 & 46,7 & 1 \\
\hline No & 62,8 & 67,2 & 53,3 & $1,21(1,01-1,45)$ \\
\hline \multicolumn{5}{|c|}{ Hipoglucemiantes orales } \\
\hline Metformina + glibenclamida & 18,1 & 12,5 & 30,0 & 1 \\
\hline Metformina + vildagliptina & 1,4 & 1,6 & 1,1 & $1,59(0,84-3,01)$ \\
\hline Vildagliptina & 1,1 & 1,0 & 1,1 & $1,42(0,60-3,32)$ \\
\hline Metformina & 67,7 & 75,0 & 52,2 & $1,60(1,18-2,17)$ \\
\hline Glibenclamida & 1,4 & 1,0 & 2,2 & $1,06(0,38-2,95)$ \\
\hline Ninguno & 10,3 & 8,9 & 13,3 & $1,25(0,82-1,90)$ \\
\hline \multicolumn{5}{|c|}{ Polifarmacia e } \\
\hline No & 39,7 & 40,6 & 37,8 & $1,04(0,88-1,22)$ \\
\hline Sí & 60,3 & 59,4 & 62,2 & 1 \\
\hline \multicolumn{5}{|c|}{ Intolerancia a los hipoglucemiantes } \\
\hline Sí & 12,1 & 9,9 & 16,7 & 1 \\
\hline No & 87,9 & 90,1 & 83,3 & $1,25(0,92-1,70)$ \\
\hline
\end{tabular}

El 99,3 \% informó que las inquietudes en relación con la medicación le fueron resultas adecuadamente por el médico en consulta y ninguno indicó que su grado de satisfacción con la atención fuera mala o regular (cuadro 3). 
Los factores que se asociaron independientemente con la adherencia farmacológica fueron: ser mujer, no tener que emplear insulina, usar metformina, haber tenido consulta con internista en el último año y presentar una HbA1c entre 6,0 y 6.
Cuadro 3. Características de los servicios de salud en los pacientes del estudio y su relación con la adherencia farmacológica

\begin{tabular}{|c|c|c|c|c|}
\hline \multirow{3}{*}{ Factores } & \multirow{3}{*}{$\begin{array}{c}\text { Total } \\
n=282 \\
\%\end{array}$} & \multicolumn{2}{|c|}{ Adherencia } & \multirow{3}{*}{$R P a(I C 95 \%)$} \\
\hline & & Si, $n=192$ & No, $n=90$ & \\
\hline & & $\%$ & $\%$ & \\
\hline \multicolumn{5}{|c|}{ Grado satisfacción del paciente con el programa } \\
\hline Buena & 59,9 & 59,9 & 60,0 & 1 \\
\hline Excelente & 40,1 & 40,1 & 40,0 & $1,00(0,85-1,18)$ \\
\hline \multicolumn{5}{|c|}{ Oportunidad en dispensación de medicación } \\
\hline No la recibió & 2,1 & 2,6 & 1,1 & $1,22(0,84-1,76)$ \\
\hline Incompleto & 15,6 & 14,6 & 17,8 & $0,93(0,73-1,18)$ \\
\hline Completo & 82,3 & 82,8 & 81,1 & 1 \\
\hline \multicolumn{5}{|c|}{ Consulta Psicología en último año } \\
\hline Sí & 55,0 & 56,8 & 51,1 & $1,08(0,91-1,27)$ \\
\hline No & 45,0 & 43,2 & 48,9 & 1 \\
\hline \multicolumn{5}{|c|}{ Consulta Trabajo Social en último año } \\
\hline Sí & 37,9 & 38,0 & 37,8 & $1,00(0,85-1,18)$ \\
\hline No & 62,1 & 62 & 62,2 & 1 \\
\hline \multicolumn{5}{|c|}{ Consulta Nutrición en último año } \\
\hline Sí & 59,9 & 57,3 & 65,6 & $0,90(0,77-1,05)$ \\
\hline No & 40,1 & 42,7 & 34,4 & 1 \\
\hline \multicolumn{5}{|c|}{$N^{\circ}$ Consultas Medicina General en último año } \\
\hline 40 más & 88,3 & 91,7 & 81,1 & $1,46(1,02-2,09)$ \\
\hline Menos de 4 & 11,7 & 8,3 & 18,9 & 1 \\
\hline \multicolumn{5}{|c|}{ Consulta Endocrinología } \\
\hline Sí & 29,4 & 25,0 & 38,9 & $0,80(0,65-0,98)$ \\
\hline No & 70,6 & 75,0 & 61,1 & 1 \\
\hline \multicolumn{5}{|c|}{ Consulta Internista en último año } \\
\hline Sí & 69,5 & 75,5 & 56,7 & $1,35(1,10-1,67)$ \\
\hline No & 30,5 & 24,5 & 43,3 & 1 \\
\hline
\end{tabular}

Al modelo explicativo final ingresaron 15 variables. Los factores que se asociaron independientemente con la adherencia farmacológica fueron: ser mujer, no tener que emplear insulina, usar metformina, haber tenido consulta con internista en el último año y presentar una HbA1c entre 6,0 y 6 (cuadro 4). 
Cuadro 4. Factores asociados a la adherencia farmacológica en diabetes mellitus tipo dos, en un programa de nefroprotección, 2017

\begin{tabular}{|c|c|c|c|c|}
\hline Factores & Categorías & $R P^{a}$ crudo & $R P^{a}$ ajustado & IC $95 \%^{b}$ \\
\hline Sexo & Mujer & 1,21 & 1,26 & $1,02-4,07$ \\
\hline \multirow{4}{*}{$\mathrm{HbA} 1 \mathrm{c}$} & $<6$ & 1,42 & 1,24 & $0,47-5,23$ \\
\hline & 6 a 6,9 & 1,70 & 1,66 & $1,37-11,80$ \\
\hline & 7 a 8,9 & 1,37 & 1,47 & $0,90-7,13$ \\
\hline & $\geq 9$ & 1 & 1 & \\
\hline Uso de insulina & No & 1,22 & 1,36 & $1,03-8,52$ \\
\hline \multirow{6}{*}{$\begin{array}{l}\text { Hipoglucemiantes } \\
\text { orales }\end{array}$} & $\begin{array}{l}\text { Metformina+ } \\
\text { Glibenclamida }\end{array}$ & 1 & 1 & \\
\hline & $\begin{array}{l}\text { Metformina+ } \\
\text { Vildagliptina }\end{array}$ & 1,59 & 1,07 & $0,08-15,90$ \\
\hline & Vildagliptina & 1,42 & 1,43 & $0,14-38,25$ \\
\hline & Metformina & 1,60 & 1,68 & $1,76-10,15$ \\
\hline & Glibenclamida & 1,06 & 1,74 & $0,26-101,08$ \\
\hline & Ninguno & 1,25 & 1,58 & $0,86-12,45$ \\
\hline $\begin{array}{l}\text { Consulta Internista en } \\
\text { último año }\end{array}$ & Si & 1,35 & 1,36 & $1,19-4,78$ \\
\hline
\end{tabular}

aRP: razón de prevalencias, ํㅣ: intervalo de confianza del $95 \%$

\section{Discusión}

Este estudio tiene similitudes y diferencias con otras investigaciones en cuanto los factores asociados a la adherencia farmacológica en diabetes mellitus; sin embargo, ninguno de ellos en el contexto de un programa de nefroprotección. Se evidenció una alta proporción de adherencia en comparación con otros estudios de Colombia y Latinoamérica, donde utilizaron también el test de Morisky Green, pues reportan porcentajes entre $52 \%$ y $57 \%$ (19-21). Esto podría estar influenciado por factores que han evidenciado estar asociados con la adherencia, pero que por su alta frecuencia en este estudio no mostraron una asociación significativa, como el grado de satisfacción de los pacientes con la atención y la oportuna dispensación de medicamentos $(20,22,23)$.

De los factores que se asociaron independientemente con la adherencia, los niveles de $\mathrm{HbA} 1 \mathrm{c}$ y el no uso de insulina, son los que muestran resultados consistentes con otros estudios y revisiones de diversos países, en los que a menor nivel de HbA1c se observa más adherencia, $(20,24,25)$. Llama la atención que se encontró mayor adherencia en quienes tuvieron niveles de $\mathrm{HbA} 1 \mathrm{c}$ entre 6,0 y 6,9, que en los que la tenían menor de 6,0 , lo cual puede estar influenciado por un comportamiento de mala adherencia que es el uso de más dosis de medicación que las prescritas, como lo reportan Paes et al. (26). La asociación de la adherencia con el control glucémico debería ser investigada más profundamente para dilucidar si se puede considerar a la adherencia farmacológica un factor causal importante en el control de la enfermedad.

Las razones que pueden explicar la asociación entre la adherencia y el no uso de insulina, son el temor de los pacientes a las punciones para la aplicación del medicamento y a los episodios de hipoglicemia, o a falsas creencias acerca de daños 
Que las citas con el médico internista se asocien independientemente con la adherencia podría ser explicado por el hecho de que este especialista puede optimizar el manejo farmacológico dado a los pacientes. de la insulina en la salud, como se ha observado en algunos estudios con enfoque cualitativo realizados en Colombia y México $(27,28)$.

Los pacientes que tomaban metformina fueron más adherentes que aquellos que la usaban en combinación con glibenclamida; esto difiere del estudio de White et al., en Inglaterra quienes reportan mayor adherencia a sulfonilureas que a metformina; sin embargo, por perfil de seguridad en Europa se suele utilizar sulfonilureas de segunda generación en lugar de glibenclamida (24). Es posible que usar únicamente metformina genere menos efectos secundarios y esto favorezca la adherencia, lo cual concuerda con que el porcentaje de pacientes adherentes fue mayor entre los que no reportaron intolerancia respecto a los que sí lo hicieron, a pesar de que no se encontró asociación.

Es necesario evaluar de forma más específica los efectos secundarios o reacciones adversas a la medicación, tanto en el ámbito asistencial como investigativo; pues los motivos por los cuales el uso de insulina o determinados hipoglucemiantes orales se asociaron con pobre adherencia, se explican en parte por efectos secundarios o reacciones adversas a la medicación (23).

Que las citas con el médico internista se asocien independientemente con la adherencia podría ser explicado por el hecho de que este especialista puede optimizar el manejo farmacológico dado a los pacientes. En los estudios revisados, esta variable solo la reporta Consuegra et al. quienes no hallan asociación entre el número de consultas con internista y la adherencia (20).

En esta investigación ser mujer se asoció independientemente con la adherencia; un estudio realizado en Estados Unidos por Bhuyan et al. en 2014 evidencia que las mujeres son menos adherentes con respecto a los hombres si se tiene en cuenta el costo de la medicación, sin embargo, en el contexto de la presente investigación, los pacientes no deben pagar nada por sus medicamentos. Se necesita realizar más investigaciones que ayuden a esclarecer los hallazgos encontrados (29).

De acuerdo a los protocolos del programa, los pacientes deben asistir a consulta con Nutrición, Psicología y Trabajo Social una vez al año. Asistir anualmente a estas consultas no se asoció con la adherencia; es posible que tener solamente una cita cada año con estos profesionales no sea suficiente para afectar la adherencia. De los estudios revisados, ninguno evaluó este tipo de variables.

Los resultados de esta investigación pueden inferirse a la población de pacientes adultos con diabetes mellitus tipo dos del programa de nefroprotección en los municipios de Pasto y Túquerres; además, son una herramienta para que en el programa de nefroprotección se implementen medidas que permitan hacer un seguimiento más estrecho a aquellos pacientes con características que se asocian a un perfil de no adherencia farmacológica.

Entre las limitaciones de la presente investigación se tienen la utilización de fuente secundaria para la recolección de varios datos, como la adherencia farmacológica, debido a que no se contaba con los recursos suficientes para aplicar directamente una escala que la midiera en los participantes; tampoco se pudieron explorar algunas variables que se han visto asociadas con pobre adherencia farmacológica, tales como depresión, consumo de alcohol y tabaquismo $(6,30)$. 
La adherencia farmacológica está influenciada por factores que no sólo dependen del paciente, sino que involucran también a los profesionales de salud, el tipo de medicación y lsu disponibilidad.
En conclusión, la adherencia farmacológica está influenciada por múltiples factores que no sólo dependen del paciente, sino que involucran también a los profesionales de salud, el tipo de medicación y la disponibilidad de la misma, además de asociarse significativamente con el control glucémico; por lo tanto, se debe fortalecer los procesos del servicio que ayuden a optimizar la prescripción y disponibilidad de la medicación, lo cual puede contribuir al mejor control de la enfermedad,.

\section{Agradecimientos}

A Cooemssanar IPS por permitir la realización de esta investigación con sus afiliados.

\section{Declaración de conflicto de intereses}

Los autores declaramos no tener conflictos de intereses. La investigación fue financiada por los autores.

\section{Bibliografía}

1. Roglic G, World Health Organization, editores. Global report on diabetes. Geneva, Switzerland: World Health Organization; 2016. 86 p.

2. World Health Organization, E-libro C. Adherencia a los tratamientos a largo plazo pruebas para la acción [Internet]. Ginebra: Organización Mundial de la Salud; 2004 [citado 23 de noviembre de 2016]. Disponible en: http://site.ebrary.com/ id/10268791

3. Edelman SV, Polonsky WH. Type 2 Diabetes in the real world: the elusive nature of glycemic control. Diabetes Care 2017;40 (11):1425-32.

4. Huber CA, Rapold R, Brüngger B, Reich O, Rosemann T. One-year adherence to oral antihyperglycemic medication and risk prediction of patient outcomes for adults with diabetes mellitus. Medicine (Baltimore) [Internet] 2016 [citado 9 de septiembre de 2018];95 (26). Disponible en: https://www.ncbi.nlm.nih.gov/pmc/ articles/PMC4937918/

5. Reynolds K, An J, Wu J, Harrison TN, Wei R, Stuart B, et al. Treatment discontinuation of oral hypoglycemic agents and healthcare utilization among patients with diabetes. Journal of Diabetes and its Complications 2016;30 (8):1443-51.

6. Krass I, Schieback P, Dhippayom T. Adherence to diabetes medication: a systematic review. Diabetic Medicine 2015;32 (6):725-37.

7. Machado-Alba JE, Medina-Morales DA, Echeverri-Cataño LF. Comparison of medication adherence in diabetes mellitus patients on human versus analogue insulins. Expert Opinion on Drug Safety 2017;16 (2):133-7.

8. Martínez Domínguez Gl, Martínez Sánchez LM, Rodríguez Gázquez M de los Á, Agudelo Vélez CA, Jiménez Jiménez JG, et al. Adherencia terapéutica y control metabólico en pacientes con diabetes mellitus tipo 2, pertenecientes a una institución hospitalaria, de la ciudad de Medellín (Colombia), año 2011. Archivos de Medicina 2014;14 (1):44-50.

9. Zambrano R, Duitama J, Posada J, Flórez J. Percepción de la adherencia a tratamientos en pacientes con factores de riesgo cardiovascular. Rev Fac Nac Salud Pública 2012;30 (2):163-74. 
10. Waari G, Mutai J, Gikunju J. Medication adherence and factors associated with poor adherence among type 2 diabetes mellitus patients on follow-up at Kenyatta National Hospital, Kenya. Pan Afr Med J [Internet] 2018 [citado 23 de septiembre de 2018];29. Disponible en: https://www.ncbi.nlm.nih.gov/pmc/articles/ PMC5987072/

11. Coordinación metodológica de la Cuenta de Alto Costo. Contenidos mínimos indispensables para la gestión del riesgo renal en un programa de atención a pacientes adultos con enfermedad renal crónica, sin terapia de reemplazo renal en Colombia. Definiciones técnicas basadas en evidencia [Internet]. 2011. Disponible en: https://www.minsalud.gov.co/sites/rid/Lists/BibliotecaDigital/RIDE/INEC/CAC/ programas ERC.pdf

12. Fondo Colombiano de Enfermedades de Alto Costo. Nefroprotección: Estrategia fundamental para evitar, controlar y retrasar el daño renal. Boletín de Información Técnica Especializada. 10 de marzo de 2016;2(2).

13. Terechenko Luhers NS, Baute Geymonat AE, Zamonsky Acuña JN. Adherencia al tratamiento en pacientes con diagnóstico de diabetes mellitus tipo ii. Biomedicina 2015;10 (1):20-33.

14. Val Jiménez A, Amorós Ballestero G, Martínez Visa P, Fernández Ferré M. Estudio descriptivo del cumplimiento del tratamiento farmacológico antihipertensivo y validación del tes Morisky y Green. Atención Primaria 1992;10 (5):767-70.

15. Wang Y, Lee J, Toh MPHS, Tang WE, Ko Y. Validity and reliability of a self-reported measure of medication adherence in patients with Type 2 diabetes mellitus in Singapore: Validation of a medication adherence measure in Singapore. Diabetic Medicine 2012;29 (9):e338-44.

16. Ministerio de Salud y Protección Social. Resolución número 4003 de 2008. 2008.

17. Zocchetti C, Consonni D, Bertazzi PA. Relationship between prevalence rate ratios and odds ratios in cross-sectional studies. Int J Epidemiol 1997;26 (1):220-3.

18. Ministerio de Salud. Normas científicas, técnicas y administrativas para la investigación en salud. Resolución Nº 008430 de 1993. 1993.

19. Bello Escamilla NV, Montoya Cáceres PA. Adherencia al tratamiento farmacológico en adultos mayores diabéticos tipo 2 y sus factores asociados. Gerokomos. junio de 2017;28 (2):73-7.

20. Consuegra Cabally DC, Diaz Cedeño MM. Factores asociados con la adherencia terapéutica en pacientes con diabetes mellitus tipo 2 atendidos en la consulta de medicina interna en un hospital de II nivel de Bogotá [Maestría en Epidemiología]. [Bogotá, Colombia]: CES; 2017.

21. De la Rubia JM, Alejandra Cerda MT. Predictores psicosociales de adherencia a la medicación en pacientes con diabetes tipo 2. Revista Iberoamericana de Psicología y Salud 2015;6 (1):19-27. 
22. Ciechanowski PS, Katon WJ, Russo JE, Walker EA. The patient-provider relationship: attachment theory and adherence to treatment in diabetes. American Journal of Psychiatry 2001;158 (1):29-35.

23. Bagonza J, Rutebemberwa E, Bazeyo W. Adherence to anti diabetic medication among patients with diabetes in eastern Uganda; a cross sectional study. BMC Health Services Research [Internet] 2015 [citado 16 de marzo de 2017];15 (1). Disponible en: http://bmchealthservres.biomedcentral.com/articles/10.1186/ s12913-015-0820-5

24. White AJS, Kellar I, Prevost AT, Kinmonth AL, Sutton S, Canny M, et al. Adherence to hypoglycaemic medication among people with type 2 diabetes in primary care. Prim Care Diabetes 2012;6 (1):27-33.

25. Doggrell SA, Warot S. The association between the measurement of adherence to anti-diabetes medicine and the $\mathrm{HbA} 1 \mathrm{c}$. International Journal of Clinical Pharmacy 2014;36 (3):488-97.

26. Paes AHP, Bakker A, Soe-Agnie CJ. Impact of Dosage Frequency on Patient Compliance. Diabetes Care 1997;20 (10):1512-7.

27. Hoyos Duque TN, Arteaga Henao MV, Muñoz Cardona M. Factores de no adherencia al tratamiento en personas con Diabetes Mellitus tipo 2 en el domicilio. La visión del cuidador familiar. Invest Educ Enferm 2011;29 (2):194-203.

28. Mercado-Ramírez MA, Escobedo-Hidalgo E, Sarabia-Avalos MÁ, Ruíz-Gómez A, González-Cerda LM, Aceves-Blanco A, et al. Factores que intervienen sobre la adherencia terapéutica en el tratamiento con insulina en pacientes diabéticos tipo II del Hospital Regional de Magdalena, Jalisco. Revista de Educación y Desarrollo. 2017;41:5-16.

29. Bhuyan SS, Shiyanbola O, Deka P, Isehunwa 00, Chandak A, Huang S, et al. The role of gender in cost-related medication nonadherence among patients with diabetes. J Am Board Fam Med 2018;31 (5):743-51.

30. Han E, Sohn HS, Lee J-Y, Jang S. Health Behaviors and Medication Adherence in Elderly Patients. American Journal of Health Promotion 2017;31 (4):278-86. 